\title{
SURGICAL NEVER EVENTS; SHOULD NEVER HAPPEN (Patient safety is a top priority)
}

\section{Thamer A Hamdan ${ }^{\circledR}$ \& Darren F Lui*}

${ }^{\circledR}$ MB,ChB, FRCS, FICS, FACS, FRCP, American Board of Neurological \& Orthopaedic Surgery, Professor of Orthopedic Surgery, Research Fellow in Saint George's University Hospital, London, Visiting Professor, Imperial College, London, UK. " MCh, FRCS Trauma \& Orthopaedics, Consultant Trauma, Orthopaedic, and Spine Surgeon, Saint George's University Hospital, London, UK.

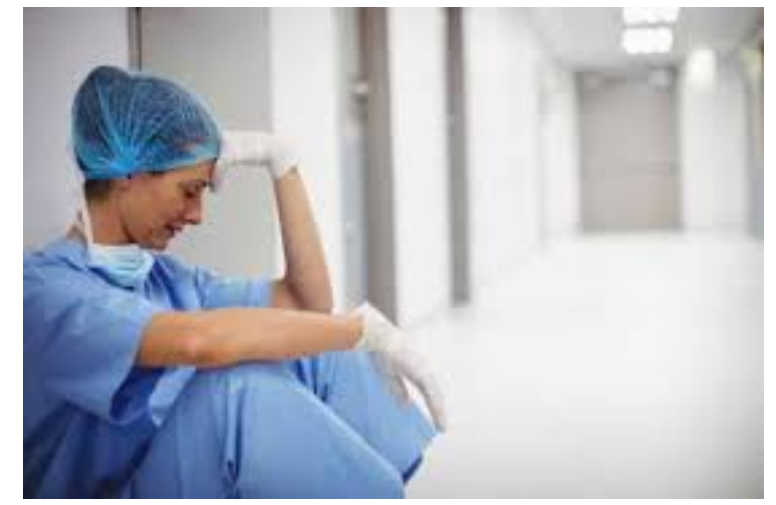

Qurgical never events are the kind of preventable mistakes that should never happen Nunder any circumstance, or it is a serious consequence to the patients and the healthcare providers.

Sadly, despite all the precautions given by (NHS) it is still occurring till now, and will continue to happen even in the best medical centers all over the globe.

Our dear patients put their trust and life in our clinical hands and judgment so we must strive to make surgery fruitful and safe as much as possible.

Certainly, it never happens if health care providers have implemented existing guidance, safety recommendations, and applies the surgical checklist given by the WHO.

The NHS recorded between April 2012 and March 2013 about 290 never events. Out of these, 130 surgical retained foreign objects including: 47 Vaginal swab, tampon, cotton wool, 34 Surgical swab, 11 Instruments, 6 Guide wire-central line, 5 Laparoscopic specimen bag (with specimen), 4 Surgical drain, 3 Glove remnant, 2 Pins, 2 Surgical needle, 2 Drill guide, 2 Guide wire-chest drain, 2 Throat pack, 2 Unknown, 2 Part of broken instrument, 1 Hypodermic needle, 1 Nasal tampon (used for a laparoscopic procedure), 1 Anterior cruciate ligament implant, 1 Guide wire-femoral line, 1 Guide wire-shoulder surgery, and 1 Silicone tubing.

There were 83 wrong site surgery including; 26 Wrong side, 21 Wrong tooth, 12 Wrong procedure, 24 Wrong implant or prosthesis including eye lenses, knee prosthesis and much more. Probably the number is higher in the United States.

Never events rarely happen if the surgeon is competent, with good skill, have wise judgment, good ethical background, and backed by a well-trained surgical team.

Surgical events are; wrong site surgery, wrong patient, wrong technique, or even wrong surgeon leaving foreign material before closing the skin, inserting wrong material such as prosthesis or implant. 
The reasons behind never events are complex and multiple. Events usually occur in the theatre and the surgeon is the first to be blamed. Almost all the causes are failure to follow the standard rules particularly the WHO checklist. The common reasons are: lack of experience from the surgeon side and the operative staff, poor surgical facilities, lack of co-operation between the team including nursing staff and the surgeons, interruption and distraction at the time of surgery, multiple surgeons operating on the same patient as in cases with multiple injuries, changing staff at the time of operation, emergency and critical patient condition, presence of difficult or complex pathology, presence of anatomical variation such as aberrant artery which may lead to a real disaster. Unfamiliarity of the surgeon may lead to confusion. Silent assistant and poor teamwork may result in actual tragedy. Any of the above reasons can create an incident.

Also, lack of concentration at the time of surgery for any reason should be avoided. Time pressure leads to hurried closure particularly when the theatre is required for awaiting emergency condition or long waiting list so the surgeon will be exhausted, have lack of concentration, and psychologically not well. In that case, it is better to stop performing the procedure and ask a colleague for help.

The mounting pressure in the medical service especially in developing countries plays a major rule in producing surgical never events, so the surgeon should realize when to stop performing procedures and no more patients when he can not manage.

Understanding the root cause and analysis are mandatory to avoid repeating the never events.

Our focus should be on the reasons behind failure rather than on the actions of the involved individual. Never events can be avoided easily if the standard guidelines are applied. There should be a strong leadership, minimally two persons to ensure perfection, application of WHO checklist, barcode scanner for blood transfusion, preoperative evaluation including the identification of blood disorders to prevent profuse bleeding, avoiding interruption, and good concentration on the field.

After the never events has occurred, various actions should be taken such as: Immediate resuscitation, reporting the incident to the hospital, apology to the patient, performing an analysis of the condition, implementing and sharing the lessons learned by procedural changes, serious actions to be taken to avoid repeating the disaster, and advices should be given to guilty workers and to be judged if they repeat the same mistake.

Finally, it is very easy to avoid falling in the trap of never events if : standard surgical rules are performed, no distraction or interruption during surgery, surgeon should be fully concentrating on his field, and well supported by a co-operative and honest surgical staff; But it is always easy to be wise after the events.

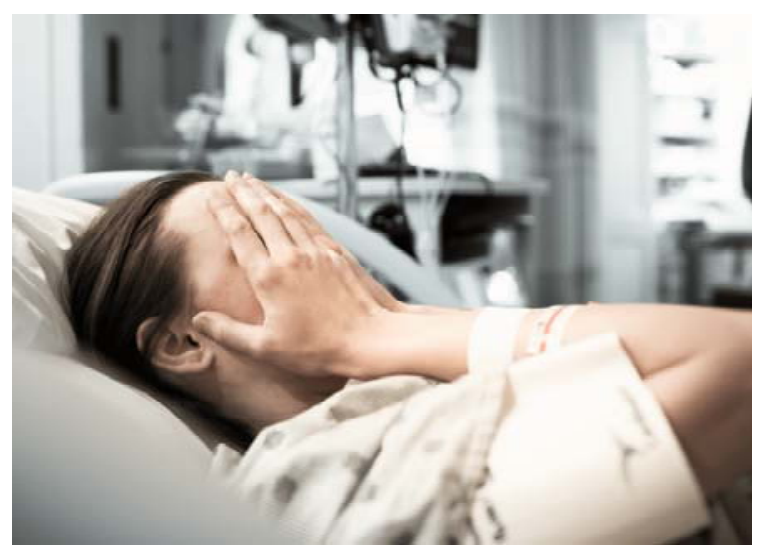

